\title{
Transmit and Receive Cooperative Cognition: Protocol Design and Stability Analysis
}

\author{
Ahmed El Shafie ${ }^{\dagger}$, Amr El-Keyi ${ }^{\dagger}$, Tamer Khattab*, Mohamed Nafie ${ }^{\dagger}$ \\ ${ }^{\dagger}$ Wireless Intelligent Networks Center (WINC), Nile University, Giza, Egypt. \\ *Electrical Engineering, Qatar University, Doha, Qatar. \\ ahmed.salahelshafie@gmail.com, aelkeyi@nileuniversity.edu.eg, tkhattab@qu.edu.qa, mnafie@nileuniversity.edu.eg
}

\begin{abstract}
In this paper, we investigate the stability of a cooperative cognitive system. We propose a cooperative secondary transmitter-receiver system (CSTR), where, the secondary transmitter (ST) and the secondary receiver (SR) increase the spectrum availability for the ST packets by relaying the unsuccessfully transmitted packets of the primary transmitter (PT). We assume receiving nodes with multipacket reception capability (MPR). We provide two inner bounds and two outer bounds on the stability region of the considered system.
\end{abstract}

Index Terms-Cognitive radio, closure, stability analysis.

\section{INTRODUCTION}

The electromagnetic radio spectrum is a precious resource, whose use is licensed by governments [1]. Regulatory bodies have come to realize that most of the time, large portions of certain licensed frequency bands remain unused. The intuitive intention behind secondary spectrum licensing is to efficiently increase the spectral usage of the network while, depending on the type of licensing, not perturbing the primary users (higher priority users). Cognitive radio (CR) systems are seen as a candidate prime solution that can significantly mitigate the current low spectral efficiency in the electromagnetic spectrum. A CR is defined as an intelligent wireless communication system that is fully aware of its environment and uses methodologies of learning and reasoning in order to dynamically adapt its transmission parameters (e.g., operating spectrum, modulation schemes, coding, and transmission power) to access portions of spectrum by exploiting the existence of spectrum holes left unused by a primary system.

The idea of cooperative cognitive transmitter has been investigated in many papers [2]-[9]. In [2], the secondary transmitter (ST) is used as a relay for the undelivered packets of the higher priority user (primary user). The secondary user controls its power for expanding the stability region of the network. In [3], an extension of the problem with multiple secondary transmitters acting as relays for the undelivered packets of the primary transmitter (PT) was investigated. Furthermore, the authors of [3] considered priority in transmission is given to the relaying queues. In [4], the authors assumed that the cognitive user transmitter will be allowed to use the channel if the PT not using the spectrum, and that the priority

Mohammed Nafie is also affiliated with the Electronics and CommunicaCROWNCOM 2013, July 08-10, Washington DC, United States of transmission is given to the relaying queue. Also, it was assumed that the secondary will decide to relay a certain fraction $f$ (adaptive admission control parameter) of the undelivered packets of the PT to minimize the ST delay subject to a power budget for the relayed primary packets. In [5], the authors characterized fundamental issues in a shared channel where the users have different priority levels. They characterized the stable-throughput region in a two user cognitive shared channel where the PT has unconditional access to the channel while the ST transmits its packets with some access probability designed based on the primary queue state. More specifically, the ST accesses the channel with probability 1 if the PT is empty, and with probability p if the PT is nonempty. In [6], the authors proposed a cluster of secondary users helping the PT with a single relaying queue accessible by all the secondary users. The authors in [7] proposed a novel channel access protocol for multiple secondary users with relaying capability in a cognitive network. The stability region of the secondary network was characterized. In [8], the authors considered a network with two primary users and one ST that relays their undelivered packets in the free time slots. In [9], the authors considered multiple PTs with a common destination and one ST with relaying capability. More specifically, the CR user senses the primary bands and sends a packet from the relaying queues until they all being emptied. Afterwards, the CR user switches to the "best" idle band, which has the maximum channel gain based on the channel conditions of the time, to send its own packets.

In this work, we propose a new cognitive system with the concept of cooperation. In the cooperative secondary transmitter-receiver system (CSTR), the cognitive system with its transmitter-receiver pair tries to utilize the periods of silence of the PT in order to increase the reliability of communication against the effect of random channel variation, i.e., channel fading, and to allow the ST to utilize the channel efficiently and effectively. More specifically, when the secondary transmitter and receiver sense the channel for empty time slots, the slots are then used to help the primary system, and/or to allow the ST's packets to be served.

We make the following contributions in this paper.

- We propose a new cooperative system (CSTR), where both the secondary transmitter and receiver are used as relays for the primary terminal.

- We design a new protocol to manage the undelivered primary packets decoding at the terminals and the channel 


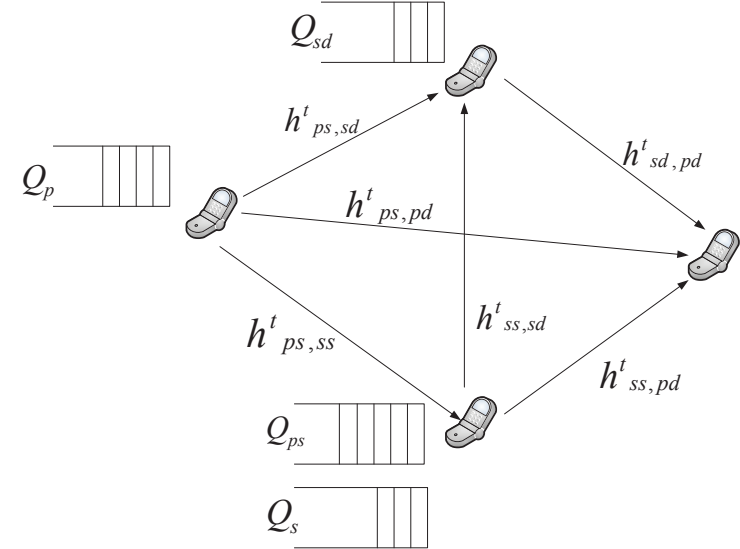

Fig. 1. Primary and secondary links and queues.

accessing of the terminals.

- We propose an access probability assigned to each queue and a controllable factor added to each relaying queue. The relaying queues' admitting factors control the arrival processes of the relaying queues and the service process of the primary queue, while the access probabilities controls the service processes of the queues.

- We consider multipacket reception (MPR) capability added to the receiving nodes.

- We provide two inner bounds and two outer bounds on the stability of the primary-secondary network, i.e., the maximum stable-throughput of the ST given certain average arrival rate to the PT queue.

For comparison purposes, we consider, in the Numerical Results section, two extra systems. The first system is the noncooperative secondary receiver (SR) with priority queueing (NCPQ) system, where the traffic of the ST is not served until the relaying queue is empty. The second system is the noncooperative SR with no priority queueing (NCNPQ) to any of the queues. Note that in NCPQ and NCNPQ systems, only the ST helps the PT and all other medium access control (MAC) operations are the same as described in the next section.

The rest of the paper is organized as follows. In the next section, we describe the system model considered in this paper. The stability analysis of the proposed system is considered in Section III. In Section IV, we provide some numerical results of the considered optimization problems in this paper, and finally, we conclude the paper in Section V.

\section{SYSTEM MODEL}

The network consists of one primary transmitter-receiver pair and one secondary transmitter-receiver pair as shown in Fig. ${ }^{1}{ }^{1}$ The ST is utilizing the spectrum resource whenever the PT is idle. We assume that the ST and SR will be able

\footnotetext{
${ }^{1}$ The proposed cognitive cooperation protocol and the theoretical development in this paper can be generalized to cognitive radio networks with more primary transmitter-receiver pairs and more secondary transmitter-receiver pairs, in which several primary transmitter-receiver pairs may choose one or more secondary transmitter-receiver pairs or the best secondary transmitterreceiver pair for cooperation.
}

to sense the channel every time slot to check whether the PT is idle or not. The cognitive system will be able to send a packet each time slot during the idle sessions of the PT. The main assumptions of the system model at both the MAC and physical (PHY) layers are discussed in this section.

\section{A. PHY Layer Assumptions}

Denote the primary source as 'ps', the primary destination as 'pd', the secondary source as 'ss', and the secondary destination as 'sd'. Let $h_{j, k}^{t}$ denote the channel gain between node $j$ and node $k$ at instant $t$, where $j, k \in\{\mathrm{ss}, \mathrm{sd}, \mathrm{ps}, \mathrm{pd}\}$, and it is distributed according to a zero mean circularly symmetric complex Gaussian random variable with variance $\sigma_{j, k}^{2}$, i.e., $\mathcal{C N}\left(0, \sigma_{j, k}^{2}\right)$. Channel gains are independent and each link is perturbed by complex additive white Gaussian noise (AWGN) with zero mean and with variance $\mathcal{N}_{\circ}$ and independent for all links. In this paper, we consider MPR channel model which can capture the effect of interference and fading at the PHY layer. Packets could survive the interference caused by concurrent transmissions if the received signalto-interference and noise ratio (SINR) exceeds the threshold required for successful decoding at the receiver, i.e., for the link between node $j$ and node $k$ the probability of successful reception of the packet from node $j$ at its receiving node $k$ when there is a concurrent transmission from node $\ell$ is given by $\bar{P}_{j, k}=\operatorname{Pr}\left\{\operatorname{SINR}>\gamma_{t h, k}\right\}$, where the superscript ' $\ell$ ' denotes the node which causes the interference (see the Appendix). Given the channel model described above, when there is no concurrent transmission, the outage probability between node $j$ and node $k$ can be calculated as follows:

$$
\begin{aligned}
\operatorname{Pr}\left\{O_{j, k}\right\}=P_{j, k} & =\operatorname{Pr}\left\{\left|h_{j, k}^{t}\right|^{2} \mathbb{P}_{j}<\mathcal{N}_{\circ} \gamma_{t h, k}\right\} \\
& =1-\exp \left(-\frac{\gamma_{t h, k} \mathcal{N}_{\circ}}{\sigma_{j, k}^{2} \mathbb{P}_{j}}\right)
\end{aligned}
$$

where $\mathbb{P}_{j}$ denotes the transmission power of node $j$ and $O_{j, k}$ is the event that the link between node $j$ and node $k$ is in outage. The SNR threshold $\gamma_{t h, k}$ is a function of different factors in the communication system; it is a function of the application, the modulation, the signal processing applied at encoder/decoder sides, error-correction codes, and many other parameters [10].

From the Appendix, the probability of packet correct reception of a transmitted packet from node $j$ to node $k$ when there is a concurrent transmission from node $\ell$ is given by

$$
\bar{P}_{j, k}=\frac{1}{1+\frac{\mathbb{P}_{\ell} \gamma_{t h, k}}{\mathbb{P}_{j}} \frac{\sigma_{\ell, k}^{2}}{\sigma_{j, k}^{2}}} \bar{P}_{j, k} .
$$

\section{B. MAC Layer Assumptions}

We assume that the PT has a buffer $Q_{\mathrm{p}}$ to store the incoming traffic packets, while the ST has two buffers, $Q_{\mathrm{s}}$ to store its own arriving traffic packets and $Q_{\mathrm{ps}}$ to store a fraction of undelivered packets of the PT. We assume all buffers are of infinite length. We consider time-slotted transmission where all packets have the same size and one time slot is sufficient for the transmission of a single packet. The arrival processes 
to the primary and the secondary queues are assumed to be independent Bernoulli processes with mean arrival rates $\lambda_{\mathrm{p}} \in$ $[0,1]$ and $\lambda_{\mathrm{s}} \in[0,1]$ packets per time slot, respectively.

In this system, denoted by $\mathcal{S}$, the ST accepts a fraction of the undelivered packets of the PT, i.e., it will decide to accept a controllable fraction to be admitted to its relaying queue in case of outage on the primary channel using an adaptive admission control parameter $f_{\mathrm{s}}$, and the SR has a buffer $Q_{\mathrm{sd}}$ to store undelivered packets of the PT in order to accept a fraction $f_{\text {sd }}$ of the undelivered packets of the PT. We assume that for successfully decoded packets by the ST and the SR, the priority of keeping the packet is one of the optimization parameters of the system defined as a binary value $\mathcal{P}$, i.e., $\mathcal{P}=1$ if the priority is assigned to the SR, and $\mathcal{P}=0$ if the priority is assigned to the ST. We assume that the acknowledgment (ACK) and negativeacknowledgement (NACK) messages initiated by the node with the higher priority of keeping are sent earlier than the messages that are sent by the lower priority one, i.e., the node with priority of keeping transmits ACKs and NACKs from $\tau_{1}$ to $\tau_{2}$ within the time slot and the other node transmits from $\tau_{2}$ to $\tau_{3}$. Note that the primary receiver (PR) sends the feedback messages over the period $\tau_{\circ}$ to $\tau_{1}{ }^{2}$ The MAC layer is assumed to obey the following transmission scheme.

- Assign the priority of keeping the undelivered PT packet to the ST or the SR at the beginning of the transmissions.

- The primary user transmits the packet at the head of its queue. If the queue is empty the time slot is free.

- If a packet is received successfully by either the PR, the ST, or the SR the packet is removed from the PT's queue (the ST or the SR needs to send an ACK if a packet is not decoded correctly by the PR in this case).

- If both the ST and SR decoded a packet correctly, the terminal which has the priority of keeping, will keep the packet and the packet will be removed from other node's buffer.

- If a packet is not received successfully by the PR, the ST, and the SR, the PT retransmits this packet in the next time slot.

- The ST and SR randomly access the channel at each sensed free time slot (ALOHA random access). ${ }^{3}$

- At each sensed empty time slot, the ST transmits a packet from its own queue with probability $p_{\mathrm{s}}$, retransmits a packet from the relaying queue with some probability $p_{\mathrm{sp}}$, or remains idle with probability $p_{i}=1-p_{\mathrm{s}}-p_{\mathrm{sp}}$. The SR attempts to retransmit the undelivered packets of the PT with probability $p_{\mathrm{sd}}$, or to remain idle with probability $1-p_{\mathrm{sd}}$.

- Packets could survive the interference caused by concurrent transmissions between the ST and the SR, if the received SINR exceeds the threshold required for

\footnotetext{
${ }^{2}$ Another approach in feedback separation can be adopted. More specifically, we can assume that the ACKs/NACKs are sent from each node at different frequency band. In this case, the node with the lower priority of keeping the primary packet will drop what it has decoded when it gets an ACK from the higher priority node.

${ }^{3}$ The sensing duration, $\tau$, is assumed to be long enough to make the assumption of perfect sensing valid (see [2], [4], [6] for a similar assumption).
}

successful decoding at the receiver.

We assume that the overhead for transmitting the ACK and NACK messages is very small compared to packet sizes. The second assumption we make is that the errors and delay in packet acknowledgement feedback is negligible, which is reasonable for short length ACK/NACK packets as low rate codes can be employed in the feedback channel [10]. In addition, nodes cannot transmit and receive at the same time which is a common assumption where terminals are equipped with single transceivers [5].

\section{Stability Analysis of The Proposed System}

Let us denote the queue sizes of the transmitting terminals at any time instant $t$ by $Q_{i}^{t}$, where $i$ reads $\mathrm{p}$ for the PT's queue, $\mathrm{s}$ for ST's queue, ps for the ST's relaying queue, and sd for the SR's relaying queue. A fundamental performance measure of a communication network is the stability of its queues. More rigourously, stability can be defined as follows [5], [10].

Definition: Queue $Q_{i} \in\left\{Q_{\mathrm{p}}, Q_{\mathrm{s}}, Q_{\mathrm{ps}}, Q_{\mathrm{sd}}\right\}$ is stable, if

$$
\lim _{t \rightarrow \infty} \operatorname{Pr}\left\{Q_{i}^{t}<y\right\}=F(y) \text { and } \lim _{y \rightarrow \infty} F(y)=1 .
$$

If the arrival and service processes are strictly stationary, then we can apply Loynes' theorem to check for stability conditions [10], [11]. This theorem states that if the arrival process and the service process of a queue are strictly stationary processes, and the average service rate is greater than the average arrival rate of the queue, then the queue is stable, otherwise the queue is unstable.

The service and arrival rates of the nodes are as follows. For terminal $i=\mathrm{p}$, given that the priority factor $\mathcal{P}=1$, i.e., the priority of keeping the packet is assigned to the SR, a packet can be served if either one of the four events is true: 1) The primary channel is in outage, the SR decides to accept the packet (with probability $f_{\mathrm{sd}}$ ), and the channel $h_{\mathrm{ps}, \mathrm{sd}}^{t}$ is not in outage; 2) the primary channel is in outage, the ST decides to accept the packet (which happens with probability $f_{\mathrm{s}}$ ) and the SR decides not to accept the packet (which happens with probability $1-f_{\mathrm{sd}}$ ), and the associated link $h_{\mathrm{ps}, \mathrm{ss}}^{t}$ is not in outage; 3 ) the primary channel is in outage, the ST and receiver both of them decide to accept the primary packet and both of them decode it correctly ${ }^{4}$; or 4) if the channel between the primary source and PR is not in outage, i.e., $\bar{O}_{\mathrm{ps}, \mathrm{pd}}^{t}$ is true $\mathrm{e}^{5}$. Due to the stationarity assumption of the channels gain, and using the outage probability formula (1), the probability of the event $\bar{O}_{\mathrm{ps}, \mathrm{pd}}^{t}$ is given by $\operatorname{Pr}\left\{\bar{O}_{\mathrm{ps}, \mathrm{pd}}^{t}\right\}=\bar{P}_{\mathrm{ps}, \mathrm{pd}}$. From the aforementioned argument, it is clear that the queue service process is stationary process and has a finite mean:

$$
\mu_{\mathrm{p}}=\bar{P}_{\mathrm{ps}, \mathrm{pd}}+P_{\mathrm{ps}, \mathrm{pd}}\left[f_{\mathrm{sd}} \bar{P}_{\mathrm{ps}, \mathrm{sd}}+\left(1-f_{\mathrm{sd}} \bar{P}_{\mathrm{ps}, \mathrm{sd}}\right) f_{\mathrm{s}} \bar{P}_{\mathrm{ps}, \mathrm{ss}}\right] .
$$

According to Loynes theorem, the stability condition of the queue $Q_{i}$ is given by

$$
\mu_{i}>\lambda_{i}
$$

\footnotetext{
${ }^{4}$ The primary packet will be buffered to the SR queue and dropped from the ST queue due to the priority of keeping assigned to the SR.

$5 \overline{(.)}$ denotes the complement of the event, i.e., $\bar{x}=1-x$.
} 
If we take the priority of keeping factor into account, the general formula of the average service rate of the PT is given by

$$
\begin{aligned}
\mu_{\mathrm{p}}=\bar{P}_{\mathrm{ps}, \mathrm{pd}}+ & P_{\mathrm{ps}, \mathrm{pd}}\left[\mathcal{P}\left(f_{\mathrm{sd}} \bar{P}_{\mathrm{ps}, \mathrm{sd}}+\left(1-f_{\mathrm{sd}} \bar{P}_{\mathrm{ps}, \mathrm{sd}}\right) f_{\mathrm{s}} \bar{P}_{\mathrm{ps}, \mathrm{ss}}\right)\right. \\
+ & \left.\overline{\mathcal{P}}\left(f_{\mathrm{s}} \bar{P}_{\mathrm{ps}, \mathrm{ss}}+\left(1-f_{\mathrm{s}} \bar{P}_{\mathrm{ps}, \mathrm{ss}}\right) f_{\mathrm{sd}} \bar{P}_{\mathrm{ps}, \mathrm{sd}}\right)\right]
\end{aligned}
$$$$
\mu_{\mathrm{p}}=\bar{P}_{\mathrm{ps}, \mathrm{pd}}+P_{\mathrm{ps}, \mathrm{pd}}\left[f_{\mathrm{sd}} \bar{P}_{\mathrm{ps}, \mathrm{sd}}+f_{\mathrm{s}} \bar{P}_{\mathrm{ps}, \mathrm{ss}}-f_{\mathrm{s}} \bar{P}_{\mathrm{ps}, \mathrm{ss}} f_{\mathrm{sd}} \bar{P}_{\mathrm{ps}, \mathrm{sd}}\right]
$$

It should be mentioned that $\mu_{\mathrm{p}}$ is independent of $\mathcal{P}$. Given that, the primary queue is empty, the ST assigns the channel to its own queue (which happens with probability $p_{\mathrm{s}}$ ), and the channel between the ST and its respective receiver is not in outage, a packet in $Q_{\mathrm{s}}$ is served if the SR is nonempty and does not access (which happens with probability $\bar{p}_{\text {sd }}$ ), or if the SR queue is empty. The probability that the primary terminal queue is empty is given by [10]

$$
\operatorname{Pr}\left\{Q_{\mathrm{p}}^{t}=0\right\}=1-\frac{\lambda_{\mathrm{p}}}{\mu_{\mathrm{p}}} .
$$

From the above argument and the expression given in (6), the mean service rate of the ST own data queue, $Q_{\mathrm{s}}$, is given by

$$
\mu_{\mathrm{s}}=\left(1-\frac{\lambda_{\mathrm{p}}}{\mu_{\mathrm{p}}}\right) \bar{P}_{\mathrm{ss}, \mathrm{sd}} p_{\mathrm{s}}\left[\operatorname{Pr}\left\{Q_{\mathrm{sd}}^{t}=0\right\}+\bar{p}_{\mathrm{sd}} \operatorname{Pr}\left\{Q_{\mathrm{sd}}^{t} \neq 0\right\}\right] .
$$

Consider now the relaying queue of the ST, $Q_{\mathrm{ps}}$. Given that the queue of the PT is empty in a time slot $t$ and the ST chooses to access the channel with the relaying queue (which happens with probability $p_{\mathrm{sp}}$ ), a packet from queue ps can be served in either one of the three following events: 1) If the SR is idle, and the channel between the ST and the PR is not in outage $\bar{O}_{\mathrm{ss}, \mathrm{pd}}^{t} ; 2$ ) if the SR does not access the channel (which happens with probability $\bar{p}_{\text {sd }}$ ), and its queue is not empty, i.e., $Q_{\mathrm{sd}}^{t} \neq 0$, and the channel between the ST and the PR is not in outage; or 3) if the queue sd in time slot $t$ is not empty, the SR accesses the channel (which happens with probability $p_{\text {sd }}$ ), and the complement of the event outage between the ST and the PR $\bar{O}_{\text {ss,pd }}^{t} \mid \mathcal{T}_{\text {sd }}$ where $\bar{O}_{j, k}^{t} \mid \mathcal{T}_{\ell}$ denotes the complement of the outage event of the channel between node $j$ and node $k$ when there is a concurrent transmission by node $\ell$. The expected value of the service process of the relaying queue $Q_{\mathrm{ps}}$ is given by

$$
\begin{aligned}
\mu_{\mathrm{ps}}=\left[\left(\operatorname { P r } \left\{Q_{\mathrm{sd}}^{t}\right.\right.\right. & \left.=0\}+\bar{p}_{\mathrm{sd}} \operatorname{Pr}\left\{Q_{\mathrm{sd}}^{t} \neq 0\right\}\right) \bar{P}_{\mathrm{ss}, \mathrm{pd}} \\
& \left.+p_{\mathrm{sd}} \operatorname{Pr}\left\{Q_{\mathrm{sd}}^{t} \neq 0\right\} \bar{P}^{s d}{ }_{\mathrm{ss}, \mathrm{pd}}\right]\left(1-\frac{\lambda_{\mathrm{p}}}{\mu_{\mathrm{p}}}\right) p_{\mathrm{sp}} .
\end{aligned}
$$

Consider now the SR queue $Q_{\text {sd }}$. Given that the queue of the PT is empty in time slot $t$, a packet from queue sd can be served if in a time slot $t$ in either one of the six following events: 1) If the SR decides to access the channel (which occur with probability $p_{\mathrm{sd}}$ ), the ST has no packets in any of its queues, i.e., $Q_{\mathrm{s}}^{t}=0$ and $Q_{\mathrm{ps}}^{t}=0$, and the complement of the event outage between the SR and the PR
$\bar{O}_{\text {sd,pd }}^{t}$; 2) if the SR decides to access the channel, the event that the ST's queues are $Q_{\mathrm{ps}}^{t} \neq 0$ and $Q_{\mathrm{s}}^{t}=0$ and it does not access the channel (which happens with probability $\bar{p}_{\mathrm{sp}}$ ), and the complement of the event outage between the SR and the PR $\bar{O}_{\mathrm{sd}, \mathrm{pd}}^{t}$; 3) if the SR decides to access the channel, the event that the ST's queues are $Q_{\mathrm{ps}}^{t}=0$ and $Q_{\mathrm{s}}^{t} \neq 0$ and it is not accessing the channel (which happens with probability $\bar{p}_{\mathrm{S}}$ ), and the complement of the event outage between the $\mathrm{SR}$ and the PR $\bar{O}_{\mathrm{sd}, \mathrm{pd}}^{t}$; 4) if the SR decides to access the channel, the event that the ST's queues are $Q_{\mathrm{ps}}^{t} \neq 0$ and $Q_{\mathrm{s}}^{t} \neq 0$ and it does not access the channel (which happens with probability $p_{i}=1-p_{\mathrm{sp}}-p_{\mathrm{s}}$ ), and the complement of the outage event between the SR and the PR $\bar{O}_{\mathrm{sd}, \mathrm{pd}}^{t}$; 5) if the SR decides to access the channel, the event that the queue $Q_{\mathrm{ps}}^{t} \neq 0$ and the ST accesses the channel (with probability $\left.p_{\mathrm{sp}}\right)$, and the complement of the outage event between the SR and the PR given a transmission between the ST and the PR $\bar{O}_{\text {sd,pd }}^{t} \mid \mathcal{T}_{\mathrm{ss}}$; or 6) if the SR decides to access the channel, the event that the queue $Q_{\mathrm{s}}^{t} \neq 0$ and the $\mathrm{ST}$ accesses the channel (with probability $p_{\mathrm{s}}$ ), and the complement of the outage event between the SR and the PR given a transmission between the ST and the SR $\bar{O}_{\text {sd,pd }}^{t} \mid \mathcal{T}_{\text {ss }}$. The expected value of the service process of $Q_{\mathrm{sd}}$ is given by

$$
\begin{aligned}
\mu_{\mathrm{sd}}=\left(1-\frac{\lambda_{\mathrm{p}}}{\mu_{\mathrm{p}}}\right) p_{\mathrm{sd}} & {\left[\left(\operatorname{Pr}\left\{Q_{\mathrm{ps}}^{t}=0, Q_{\mathrm{s}}^{t}=0\right\}\right.\right.} \\
+\bar{p}_{\mathrm{sp}} \operatorname{Pr}\left\{Q_{\mathrm{ps}}^{t} \neq 0, Q_{\mathrm{s}}^{t}=0\right\}+\bar{p}_{\mathrm{s}} \operatorname{Pr}\left\{Q_{\mathrm{ps}}^{t}=0, Q_{\mathrm{s}}^{t} \neq 0\right\} & \left.+p_{i} \operatorname{Pr}\left\{Q_{\mathrm{ps}}^{t} \neq 0, Q_{\mathrm{s}}^{t} \neq 0\right\}\right) \bar{P}_{\mathrm{sd}, \mathrm{pd}} \\
& \left.+\left(p_{\mathrm{sp}} \operatorname{Pr}\left\{Q_{\mathrm{ps}}^{t} \neq 0\right\}+p_{\mathrm{s}} \operatorname{Pr}\left\{Q_{\mathrm{s}}^{t} \neq 0\right\}\right) \bar{P}_{\mathrm{sd}, \mathrm{pd}}^{s s}\right] .
\end{aligned}
$$

The arrival process to the relaying queue, $Q_{\mathrm{ps}}$, can be described as follows. Given that $\mathcal{P}=1$, the PT's queue is not empty, i.e., $\left\{Q_{\mathrm{p}}^{t}>0\right\}$, the associated channel between PT and PR is in outage, the ST decides to accept the packet, and the channel between the PT and the ST is not in outage, hence, the arrival to $Q_{\mathrm{ps}}$ is either one of the following events: 1) The event that the SR decides to accept the packet from the PT, and the associated channel between the PT and the SR is in outage; or 2) if the SR decides not to accept the packet. The process is stationary and the expected value of the arrival process is expressed as

$$
\lambda_{\mathrm{ps}}=\frac{\lambda_{\mathrm{p}}}{\mu_{\mathrm{p}}} P_{\mathrm{ps}, \mathrm{pd}}\left(1-f_{\mathrm{sd}} \bar{P}_{\mathrm{ps}, \mathrm{sd}}\right) f_{\mathrm{s}} \bar{P}_{\mathrm{ps}, \mathrm{ss}} .
$$

Adding the keeping priority factor, the mean arrival rate of the queue $Q_{\mathrm{ps}}$ is then given by

$$
\lambda_{\mathrm{ps}}=\frac{\lambda_{\mathrm{p}}}{\mu_{\mathrm{p}}} P_{\mathrm{ps}, \mathrm{pd}}\left(1-\mathcal{P} f_{\mathrm{sd}} \bar{P}_{\mathrm{ps}, \mathrm{sd}}\right) f_{\mathrm{s}} \bar{P}_{\mathrm{ps}, \mathrm{ss}} .
$$

The arrival process to $Q_{\mathrm{sd}}$ can be described as follows. The event that the primary has packets, i.e., $\left\{Q_{\mathrm{p}}^{t}>0\right\}$, the SR decides to accept a packet from the PT $W_{\mathrm{sd}}^{t}$, the channel between the PT and the SR is connected, and the associated 
channel to the primary terminal-PR is in outage. The process can be modeled as

$$
\mathcal{A}_{\mathrm{sd}}^{t}=1\left[W_{\mathrm{sd}}^{t} \bigcap\left\{Q_{\mathrm{p}}^{t}>0\right\} \bigcap O_{\mathrm{ps}, \mathrm{pd}}^{t} \bigcap \bar{O}_{\mathrm{ps}, \mathrm{sd}}^{t}\right] .
$$

The process is stationary and the expected value of the arrival process to the queue $Q_{\mathrm{sd}}$ is expressed as

$$
\lambda_{\mathrm{sd}}=f_{\mathrm{sd}} P_{\mathrm{ps}, \mathrm{pd}} \bar{P}_{\mathrm{ps}, \mathrm{sd}} \frac{\lambda_{\mathrm{p}}}{\mu_{\mathrm{p}}} .
$$

If we involve $\mathcal{P}$, the mean arrival rate of the SR queue is given by

$$
\lambda_{\mathrm{sd}}=\frac{\lambda_{\mathrm{p}}}{\mu_{\mathrm{p}}} P_{\mathrm{ps}, \mathrm{pd}}\left(1-\overline{\mathcal{P}} f_{\mathrm{s}} \bar{P}_{\mathrm{ps}, \mathrm{ss}}\right) f_{\mathrm{sd}} \bar{P}_{\mathrm{ps}, \mathrm{sd}} .
$$

Since the mean service rates at nodes s, ps and sd depend on each others queue size, these queues are called interacting queues, and consequently the rates of the individual departure processes cannot be computed directly. In order to overcome this problem, we utilize the idea of stochastic dominance, which has been applied before to analyze interacting queues in ALOHA systems [5], [10], [12], [13], to obtain inner bounds on the stability region. For the outer bounds, we upper bound the queues service rates such that the service rates of the queues become decoupled.

\section{A. CSTR: Inner Bound}

The inner bound is the union over two inner bounds based on two dominant systems.

1) First Dominant System: In this system designated as $\mathcal{S}_{1}$, $Q_{\text {sd }}$ and $Q_{\mathrm{s}}$ send dummy packets when their queues are empty, and the $Q_{\mathrm{ps}}$ behaves exactly as it would in the original system $\mathcal{S}$. Now, we can write down the service and arrival rates of the interacting queues, i.e., $Q_{\mathrm{s}}, Q_{\mathrm{ps}}$ and $Q_{\mathrm{sd}}$ as follows. The mean service rates of $Q_{\mathrm{s}}$ and $Q_{\mathrm{ps}}$ are given by

$$
\begin{gathered}
\mu_{\mathrm{s}}=\left(1-\frac{\lambda_{\mathrm{p}}}{\mu_{\mathrm{p}}}\right)\left(1-P_{\mathrm{ss}, \mathrm{sd}}\right) p_{\mathrm{s}} \bar{p}_{\mathrm{sd}}, \\
\mu_{\mathrm{ps}}=\left(1-\frac{\lambda_{\mathrm{p}}}{\mu_{\mathrm{p}}}\right) p_{\mathrm{sp}}\left[\bar{p}_{\mathrm{sd}} \bar{P}_{\mathrm{ss}, \mathrm{pd}}+p_{\mathrm{sd}} \bar{P}^{s d}{ }_{\mathrm{ss}, \mathrm{pd}}\right] .
\end{gathered}
$$

The probability $\operatorname{Pr}\left\{Q_{\mathrm{ps}}=0\right\}$ is given by

$$
\operatorname{Pr}\left\{Q_{\mathrm{ps}}=0\right\}=1-\frac{\lambda_{\mathrm{ps}}}{\mu_{\mathrm{ps}}} \text {. }
$$

Therefore,

$$
\begin{aligned}
\mu_{\mathrm{sd}}=\left(1-\frac{\lambda_{\mathrm{p}}}{\mu_{\mathrm{p}}}\right) p_{\mathrm{sd}}\left[\left(\bar{p}_{\mathrm{s}}\left(1-\frac{\lambda_{\mathrm{ps}}}{\mu_{\mathrm{ps}}}\right)+\right.\right. & \left.p_{i} \frac{\lambda_{\mathrm{ps}}}{\mu_{\mathrm{ps}}}\right) \bar{P}_{\mathrm{sd}, \mathrm{pd}} \\
& \left.+\left(p_{\mathrm{sp}} \frac{\lambda_{\mathrm{ps}}}{\mu_{\mathrm{ps}}}+p_{\mathrm{s}}\right) \bar{P}^{s s}{ }_{\mathrm{sd}, \mathrm{pd}}\right] .
\end{aligned}
$$

According to the construction of the dominant system $\mathcal{S}_{1}$, it is easy to see that the queues of the dominant system are never less than those of the original system, provided they are both initialized identically (with the same initial conditions for queue sizes in both the original and dominant system). This is because, in the dominant system $\mathcal{S}_{1}$, the SR transmits dummy packets even if it does not have any packets in its queue, and therefore interferes with ST in all cases that it would in the original system. Therefore, if the queues at all nodes are stable in the dominant system, then the corresponding queues in the original system must be stable. The first inner bound $\mathcal{R}\left(\mathcal{S}_{1}\right)$ which is based on $\mathcal{S}_{1}$ is given by the closure of the rate pairs $\left(\lambda_{\mathrm{p}}, \lambda_{\mathrm{s}}\right)$ constrained by equations shown above as $f_{\mathrm{s}}, f_{\mathrm{sd}}, p_{\mathrm{s}}$, $p_{\mathrm{sp}}$, and $p_{\mathrm{sd}}$ vary over [0,1], and $\mathcal{P}$ varies over $\{0,1\}$ [5], [13]. For a fixed $\lambda_{\mathrm{p}}$, the maximum stable arrival rate to the ST's queue is given by the following optimization problem (as in [5], [10]):

$$
\begin{gathered}
\max _{p_{\mathrm{s}}, p_{\mathrm{sp}}, p_{\mathrm{pd}}, f_{\mathrm{s}}, f_{\mathrm{sd}}, \mathcal{P}} \quad \lambda_{\mathrm{s}}=\mu_{\mathrm{s}} \\
\text { s.t. } 0 \leq p_{\mathrm{s}}, p_{\mathrm{sp}}, p_{\mathrm{sd}}, f_{\mathrm{s}}, f_{\mathrm{sd}} \leq 1, \mathcal{P} \in\{0,1\}, p_{\mathrm{s}}+p_{\mathrm{sp}} \leq 1 \\
\lambda_{\mathrm{p}} \leq \mu_{\mathrm{p}}, \lambda_{\mathrm{ps}} \leq \mu_{\mathrm{ps}}, \lambda_{\mathrm{sd}} \leq \mu_{\mathrm{sd}} .
\end{gathered}
$$

2) Second Dominant System: The second dominant system is designated as $\mathcal{S}_{2}$, where the $\mathrm{ST}$ is the one that sends dummy packets from $Q_{\mathrm{s}}$ and $Q_{\mathrm{ps}}$, i.e., $\operatorname{Pr}\left\{Q_{\mathrm{s}}=0\right\}=\operatorname{Pr}\left\{Q_{\mathrm{ps}}=0\right\}=0$, and the SR behaves exactly as it would in the original system $\mathcal{S}$. The mean service rate of $Q_{\mathrm{sd}}$ is given by

$$
\mu_{\mathrm{sd}}=\left(1-\frac{\lambda_{\mathrm{p}}}{\mu_{\mathrm{p}}}\right) p_{\mathrm{sd}}\left[p_{i} \bar{P}_{\mathrm{sd}, \mathrm{pd}}+\left(p_{\mathrm{sp}}+p_{\mathrm{s}}\right) \overline{P s s}_{\mathrm{sd}, \mathrm{pd}}\right] \text {. }
$$

The probability that $Q_{\text {sd }}$ is empty is given by

$$
\operatorname{Pr}\left\{Q_{\mathrm{sd}}=0\right\}=1-\frac{\lambda_{\text {sd }}}{\mu_{\mathrm{sd}}} .
$$

Thus, the mean service rate of the ST's queues are given by

$$
\begin{gathered}
\mu_{\mathrm{s}}=\left(1-\frac{\lambda_{\mathrm{p}}}{\mu_{\mathrm{p}}}\right) \bar{P}_{\mathrm{ss}, \mathrm{sd}} p_{\mathrm{s}}\left[1-p_{\mathrm{sd}} \frac{\lambda_{\mathrm{sd}}}{\mu_{\mathrm{sd}}}\right] \\
\mu_{\mathrm{ps}}=\left(1-\frac{\lambda_{\mathrm{p}}}{\mu_{\mathrm{p}}}\right) p_{\mathrm{sp}}\left[\left(1-p_{\mathrm{sd}} \frac{\lambda_{\mathrm{sd}}}{\mu_{\mathrm{sd}}}\right) \bar{P}_{\mathrm{ss}, \mathrm{pd}}+p_{\mathrm{sd}} \frac{\lambda_{\mathrm{sd}}}{\mu_{\mathrm{sd}}} \bar{P}_{\mathrm{ss}, \mathrm{pd}}\right] .
\end{gathered}
$$

The second inner bound for the stable-throughput region of the CSTR system, $\mathcal{R}\left(\mathcal{S}_{2}\right)$, which is based on the dominant system $\mathcal{S}_{2}$, can be obtained by formulating a constrained optimization problem similar to that discussed above for the first dominant system, where we fix $\lambda_{\mathrm{p}}$ and maximize $\lambda_{\mathrm{s}}$ as $f_{\mathrm{s}}, f_{\mathrm{ps}}, p_{\mathrm{s}}, p_{\mathrm{sp}}$ and $p_{\mathrm{sd}}$ vary over $[0,1]$ and $\mathcal{P}$ varies over $\{0,1\} .{ }^{6}$

\section{B. CSTR: Outer Bound}

Here we provide two outer bounds for CSTR.

1) First Outer Bound: The first outer bound for the CSTR, denoted by $\mathcal{S}_{1}^{(o)}$, can be obtained by upper bounding the joint probability identities and using Bayes' theorem [7]. More specifically,

$$
\begin{aligned}
\operatorname{Pr}\left\{Q_{\mathrm{sd}}^{t}=0\right\}+\bar{p}_{\mathrm{sd}} \operatorname{Pr}\left\{Q_{\mathrm{sd}}^{t} \neq 0\right\} & \leq \operatorname{Pr}\left\{Q_{\mathrm{sd}}^{t}=0\right\}+\operatorname{Pr}\left\{Q_{\mathrm{sd}}^{t} \neq 0\right\} \\
& =1,
\end{aligned}
$$

\footnotetext{
${ }^{6}$ The optimization problems of the first and second dominant systems are solved numerically using MatLab optimization toolbox. Since the problems are nonconvex, the solver produces a locally optimum solution. To increase the likelihood of obtaining the global optimum, the program is run many times, say 10000 times, with different initializations of the optimization variables.
} 
and

$$
\begin{aligned}
\left(\operatorname{Pr}\left\{Q_{\mathrm{sd}}^{t}=0\right\}\right. & \left.+\bar{p}_{\mathrm{sd}} \operatorname{Pr}\left\{Q_{\mathrm{sd}}^{t} \neq 0\right\}\right) \bar{P}_{\mathrm{ss}, \mathrm{pd}} \\
& +p_{\mathrm{sd}} \operatorname{Pr}\left\{Q_{\mathrm{sd}}^{t} \neq 0\right\} \bar{P}_{\mathrm{ss}, \mathrm{pd}} \leq \bar{P}_{\mathrm{ss}, \mathrm{pd}} .
\end{aligned}
$$

Based on Bayes' theorem, we have

$$
\begin{gathered}
\operatorname{Pr}\{a, b\}=\operatorname{Pr}\{a \mid b\} \operatorname{Pr}\{b\} \leq \operatorname{Pr}\{b\} \\
\text { or } \\
\operatorname{Pr}\{a, b\}=\operatorname{Pr}\{b \mid a\} \operatorname{Pr}\{a\} \leq \operatorname{Pr}\{a\}
\end{gathered}
$$

where $a$ and $b$ are any two arbitrary events. We can upper bound the following quantities in formula (9):

$$
\begin{aligned}
& \operatorname{Pr}\left\{Q_{\mathrm{ps}}^{t}=0, Q_{\mathrm{s}}^{t}=0\right\} \leq \operatorname{Pr}\left\{Q_{\mathrm{ps}}^{t}=0\right\}, \\
& \operatorname{Pr}\left\{Q_{\mathrm{ps}}^{t} \neq 0, Q_{\mathrm{s}}^{t}=0\right\} \leq \operatorname{Pr}\left\{Q_{\mathrm{ps}}^{t} \neq 0\right\}, \\
& \operatorname{Pr}\left\{Q_{\mathrm{ps}}^{t}=0, Q_{\mathrm{s}}^{t} \neq 0\right\} \leq \operatorname{Pr}\left\{Q_{\mathrm{ps}}^{t}=0\right\}, \\
& \operatorname{Pr}\left\{Q_{\mathrm{ps}}^{t} \neq 0, Q_{\mathrm{s}}^{t} \neq 0\right\} \leq \operatorname{Pr}\left\{Q_{\mathrm{ps}}^{t} \neq 0\right\} .
\end{aligned}
$$

Based on the above facts, the mean service rates of the ST's queues can be upper bounded as follows:

$$
\mu_{\mathrm{s}} \leq\left(1-\frac{\lambda_{\mathrm{p}}}{\mu_{\mathrm{p}}}\right) \bar{P}_{\mathrm{ss}, \mathrm{sd}} p_{\mathrm{s}}, \quad \mu_{\mathrm{ps}} \leq\left(1-\frac{\lambda_{\mathrm{p}}}{\mu_{\mathrm{p}}}\right) p_{\mathrm{sp}} \bar{P}_{\mathrm{ss}, \mathrm{pd}}
$$

Therefore, the mean service rate of $Q_{\mathrm{sd}}$ is upper bounded as follows:

$$
\begin{aligned}
\mu_{\mathrm{sd}} \leq\left(1-\frac{\lambda_{\mathrm{p}}}{\mu_{\mathrm{p}}}\right) p_{\mathrm{sd}} & {\left[\left(\left(1-\frac{\lambda_{\mathrm{ps}}}{\mu_{\mathrm{ps}}}\right)+\bar{p}_{\mathrm{sp}} \frac{\lambda_{\mathrm{ps}}}{\mu_{\mathrm{ps}}}+\bar{p}_{\mathrm{s}}\left(1-\frac{\lambda_{\mathrm{ps}}}{\mu_{\mathrm{ps}}}\right)\right.\right.} \\
+ & \left.\left.p_{i} \frac{\lambda_{\mathrm{ps}}}{\mu_{\mathrm{ps}}}\right) \bar{P}_{\mathrm{sd}, \mathrm{pd}}+\left(p_{\mathrm{sp}} \frac{\lambda_{\mathrm{ps}}}{\mu_{\mathrm{ps}}}+p_{\mathrm{s}}\right) \bar{P}^{s s}{ }_{\mathrm{sd}, \mathrm{pd}}\right] .
\end{aligned}
$$

When the inequalities hold, the queues are not interacting anymore and therefore we can obtain the outer bound by solving a constrained optimization problem to get the closure $\left(\lambda_{\mathrm{p}}, \lambda_{\mathrm{s}}\right)$. The optimization problem is similar to (19).

2) Second Outer Bound: Another outer bound which can be stated analytically is obtained as follows. Using (5):

$$
\begin{aligned}
\mu_{\mathrm{p}} & =\bar{P}_{\mathrm{ps}, \mathrm{pd}}+P_{\mathrm{ps}, \mathrm{pd}}\left[f_{\mathrm{sd}} \bar{P}_{\mathrm{ps}, \mathrm{sd}}+f_{\mathrm{s}} \bar{P}_{\mathrm{ps}, \mathrm{ss}}-f_{\mathrm{s}} \bar{P}_{\mathrm{ps}, \mathrm{ss}} f_{\mathrm{sd}} \bar{P}_{\mathrm{ps}, \mathrm{sd}}\right] \\
& \leq \bar{P}_{\mathrm{ps}, \mathrm{pd}}+P_{\mathrm{ps}, \mathrm{pd}}\left(1-P_{\mathrm{ps}, \mathrm{sd}} P_{\mathrm{ps}, \mathrm{ss}}\right)=1-P_{\mathrm{ps}, \mathrm{pd}} P_{\mathrm{ps}, \mathrm{sd}} P_{\mathrm{ps}, \mathrm{ss}},
\end{aligned}
$$

$$
\mu_{\mathrm{s}} \leq\left(1-\frac{\lambda_{\mathrm{p}}}{\mu_{\mathrm{p}}}\right) \bar{P}_{\mathrm{ss}, \mathrm{sd}} p_{\mathrm{s}} \leq\left(1-\frac{\lambda_{\mathrm{p}}}{\mu_{\mathrm{p}}}\right) \bar{P}_{\mathrm{ss}, \mathrm{sd}} .
$$

When the inequality (28) holds and applying Loynes' law

$$
\lambda_{\mathrm{s}}<\mu_{\mathrm{s}} \leq\left(1-\frac{\lambda_{\mathrm{p}}}{1-P_{\mathrm{ps}, \mathrm{pd}} P_{\mathrm{ps}, \mathrm{sd}} P_{\mathrm{ps}, \mathrm{ss}}}\right) \bar{P}_{\mathrm{ss}, \mathrm{sd}}
$$

Denote the second outer bound as $\mathcal{S}_{2}^{(o)}$. The outer bound can be characterized by the rate pairs

$$
\mathcal{R}\left(\mathcal{S}_{2}^{(o)}\right)=\left\{\left(\lambda_{\mathrm{p}}, \lambda_{\mathrm{s}}\right): \frac{\lambda_{\mathrm{s}}}{\bar{P}_{\mathrm{ss}, \mathrm{sd}}}+\frac{\lambda_{\mathrm{p}}}{1-P_{\mathrm{ps}, \mathrm{pd}} P_{\mathrm{ps}, \mathrm{sd}} P_{\mathrm{ps}, \mathrm{ss}}}<1\right\} .
$$

The outer bound, $\mathcal{S}^{(o)}$, of the CSTR is the intersection of the two outer bounds, i.e., $\mathcal{R}\left(\mathcal{S}^{(o)}\right)=\mathcal{R}\left(\mathcal{S}_{1}^{(o)}\right) \bigcap \mathcal{R}\left(\mathcal{S}_{2}^{(o)}\right)$. Note that since the service rates of the queues in $\mathcal{S}_{1}^{(o)}$ are upper bounded to obtain the mean service rates of the queues in $\mathcal{S}_{2}^{(o)}$ (see (28) to (30)), therefore, $\mathcal{R}\left(\mathcal{S}_{1}^{(o)}\right)$ is contained inside $\mathcal{R}\left(\mathcal{S}_{2}^{(o)}\right)$, i.e., $\mathcal{R}\left(\mathcal{S}_{1}^{(o)}\right) \bigcap \mathcal{R}\left(\mathcal{S}_{2}^{(o)}\right)=\mathcal{R}\left(\mathcal{S}_{1}^{(o)}\right)$.

\section{Numerical Results}

In this section we provide the solution of the optimization problems considered in this paper. The inner (the union of the dominant systems stability regions) and the outer (intersection of the proposed outer bounds) bounds of the CSTR are depicted in Fig. 2. The parameters used to generate the figure are: $P_{\mathrm{ps}, \mathrm{pd}}=0.2 P_{\mathrm{ss}, \mathrm{sd}}=0.2, P_{\mathrm{ps}, \mathrm{ss}}=0.08$, $P_{\mathrm{ss}, \mathrm{pd}}=0.08, P_{\mathrm{sd}, \mathrm{pd}}=0.02, P_{\mathrm{ps}, \mathrm{sd}}=0.02, P_{\mathrm{ss}, \mathrm{pd}}^{\mathrm{sd}}=0.54$, and $P_{\text {sd,pd }}^{\mathrm{ss}}=0.51$. It can be seen that the inner and outer bounds almost overlap for the parameters used. This might indicate that the bounds are tight.

A comparison between the proposed system, NCNPQ and NCPQ is depicted in Fig. 3. The parameters used to generate this figure are: $P_{\mathrm{ps}, \mathrm{pd}}=0.4, P_{\mathrm{ss}, \mathrm{sd}}=0.2, P_{\mathrm{ps}, \mathrm{ss}}=0.08$, $P_{\mathrm{ss}, \mathrm{pd}}=0.08, P_{\mathrm{sd}, \mathrm{pd}}=0.02, P_{\mathrm{ps}, \mathrm{sd}}=0.02, P_{\mathrm{ss}, \mathrm{pd}}^{\mathrm{sd}}=0.54$, and $P_{\text {sd,pd }}^{\mathrm{ss}}=0.51$. Note that NCPQ and NCNPQ are coincide for the used parameters. It should be mentioned that if the link of the primary is always in outage with probability 1 , i.e., there is no direct link between the PT and PR (the PT's packets will never be served if there is no cooperation, i.e., $\mu_{\mathrm{p}}=\bar{P}_{\mathrm{ps} \text {,pd }}=0$ ), the CSTR performance will significantly overcome the maximum stable throughput of (NCPQ) and (NCNPQ) as seen in Fig. 4. The parameters used to generate the figure are: $P_{\mathrm{ps}, \mathrm{pd}}=1, P_{\mathrm{ss}, \mathrm{sd}}=0.2, P_{\mathrm{ps}, \mathrm{ss}}=0.8, P_{\mathrm{ss}, \mathrm{pd}}=$ $0.8, P_{\mathrm{sd}, \mathrm{pd}}=0.02, P_{\mathrm{ps}, \mathrm{sd}}=0.02, P_{\mathrm{ss}, \mathrm{pd}}^{\mathrm{sd}}=0.9$, and $P_{\text {sd,pd }}^{\mathrm{ss}}=0.51$.

From the figures, the envelope of the stability is a monotonically decreasing with the mean arrival rate of the PT, $\lambda_{\mathrm{p}}$. This is because as the the primary arrival rate increases the probability of the primary queue to be empty vanishes and the secondary queue will not be able to access the channel. It is also noted that the feasible range of the primary arrival rate expands due to cooperation.

\section{Conclusion}

In this paper, we have addressed the impact of cooperative cognition on the stability region of a network composing of one primary transmitter-receiver pair and one secondary transmitter-receiver pair. We have investigated the maximum stable-throughput of the CSTR. In CSTR, the cognitive transmitter and receiver sense the channel for idle channel resources and exploit them to either relay the undelivered packets of the PT or serve the ST own data traffic. We have provided two inner and outer bounds on the stability region of the network.

\section{APPENDIX}

Under Rayleigh fading channels, the outage probability between terminal $j$ and terminal $k$ when there is a transmission 


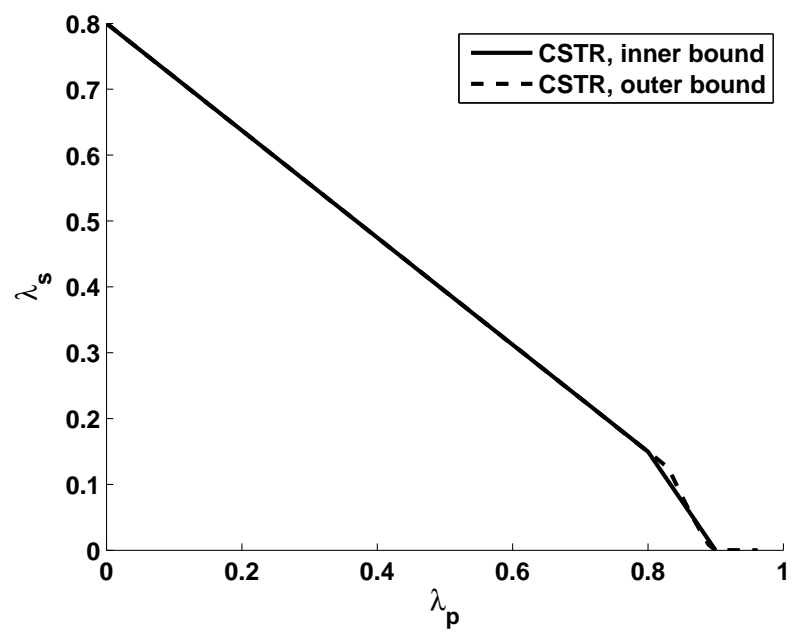

Fig. 2. Inner and outer bounds for CSTR: $P_{\mathrm{ps}, \mathrm{pd}}=0.2 P_{\mathrm{ss}, \mathrm{sd}}=0.2$, $P_{\mathrm{ps}, \mathrm{ss}}=0.08, P_{\mathrm{ss}, \mathrm{pd}}=0.08, P_{\mathrm{sd}, \mathrm{pd}}=0.02, P_{\mathrm{ps}, \mathrm{sd}}=0.02, P_{\mathrm{ss}, \mathrm{pd}}^{\mathrm{sd}}=$ 0.54 , and $P_{\text {sd,pd }}^{\mathrm{ss}}=0.51$.

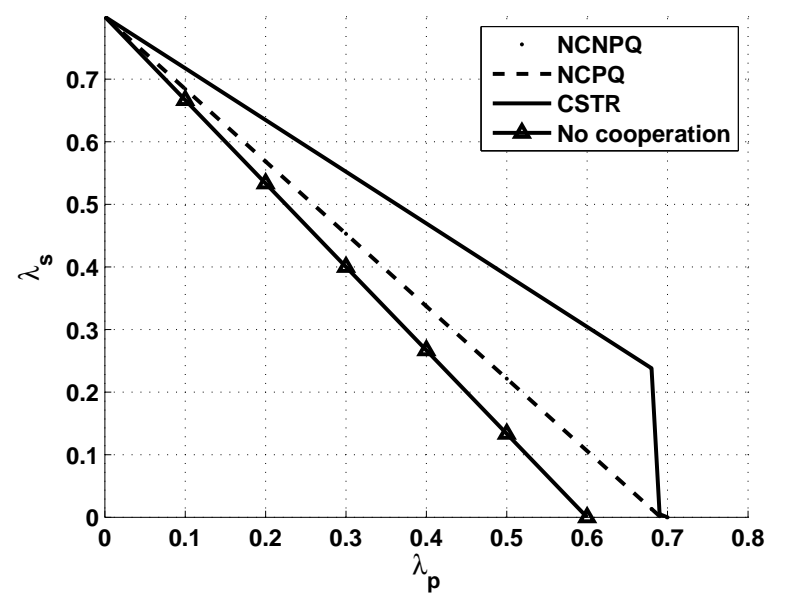

Fig. 3. Comparison between the maximum stable-throughput of the CSTR, NCPQ, NCNPQ, and the no cooperation case: $P_{\mathrm{ps}, \mathrm{pd}}=0.4, P_{\mathrm{ss}, \mathrm{sd}}=0.2$, $P_{\mathrm{ps}, \mathrm{ss}}=0.08, P_{\mathrm{ss}, \mathrm{pd}}=0.08, P_{\mathrm{sd}, \mathrm{pd}}=0.02, P_{\mathrm{ps}, \mathrm{sd}}=0.02, P^{\mathrm{sd}}{ }_{\mathrm{ss}, \mathrm{pd}}=$ 0.54 , and $P_{\text {sd,pd }}^{\mathrm{ss}}=0.51$. Note that NCPQ and NCNPQ are coincide for the used parameters.

caused by node $i[5]$ is given by

$$
\begin{aligned}
P_{j, k}^{\ell}=\operatorname{Pr}\left\{O_{j, k} \mid \mathcal{T}_{\ell}\right\} & =\operatorname{Pr}\left\{\frac{\mathbb{P}_{j}\left|h_{j, k}\right|^{2}}{\mathbb{P}_{\ell}\left|h_{\ell, k}\right|^{2}+\mathcal{N}_{\circ}} \leq \gamma_{t h, k}\right\} \\
& =1-\frac{1}{1+\frac{\mathbb{P}_{\ell} \gamma_{t h, k}}{\mathbb{P}_{j}} \frac{\sigma_{\ell, k}^{2}}{\sigma_{j, k}^{2}}} \exp \left(-\frac{\gamma_{t h, k} \mathcal{N}_{\circ}}{\mathbb{P}_{j} \sigma_{j, k}^{2}}\right)
\end{aligned}
$$

where $\left\{\mathcal{T}_{\ell}\right\}$ is the event that the terminal $\ell$ is transmitting a packet. After some mathematical manipulations, it can shown that the probability of correct packet reception in case of interference is given by

$$
\bar{P}_{j, k}=\frac{1}{1+\frac{\mathbb{P}_{\ell} \gamma_{t h, k}}{\mathbb{P}_{j}} \frac{\sigma_{\ell, k}^{2}}{\sigma_{j, k}^{2}}} \bar{P}_{j, k} .
$$

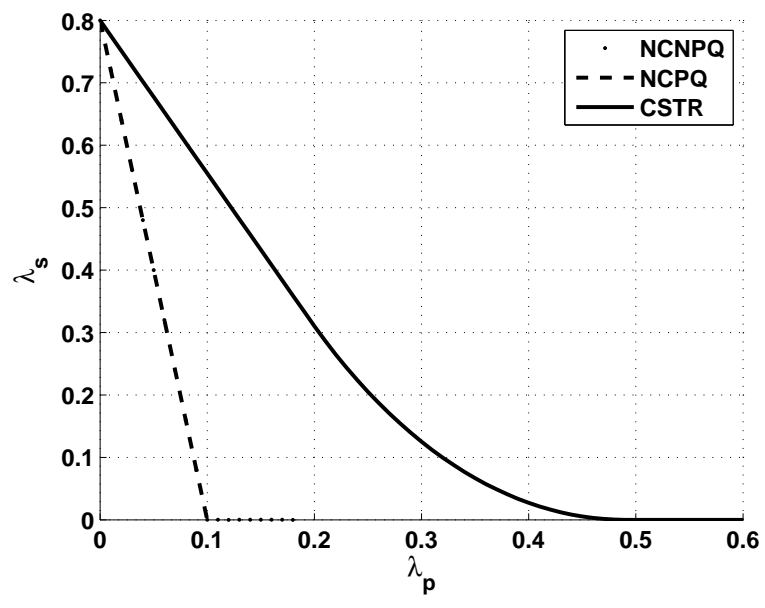

Fig. 4. Worst case comparison between the maximum stable-throughput of the CSTR, NCPQ and NCNPQ systems: $P_{\mathrm{ps}, \mathrm{pd}}=1, P_{\mathrm{ss}, \mathrm{sd}}=0.2, P_{\mathrm{ps}, \mathrm{ss}}=$ $0.8, P_{\mathrm{ss}, \mathrm{pd}}=0.8, P_{\mathrm{sd}, \mathrm{pd}}=0.02, P_{\mathrm{ps}, \mathrm{sd}}=0.02, P_{\mathrm{ss}, \mathrm{pd}}^{\mathrm{sd}}=0.9$, and $P^{\mathrm{ss}}$ sd,pd $=0.51$. Note that NCPQ and NCNPQ are coincide for the used parameters. The mean service rate of the primary queue, in case of no cooperation, is zero, i.e., $\bar{P}_{\mathrm{ps}, \mathrm{pd}}=0$.

\section{ACKNOWLEDGEMENT}

This research work is funded by Qatar National Research Fund (QNRF) under grant number NPRP 09-1168-2-455.

\section{REFERENCES}

[1] S. Haykin, "Cognitive radio: brain-empowered wireless communications," IEEE J. Sel. Areas Commun., vol. 23, no. 2, pp. 201-220, Feb. 2005.

[2] O. Simeone, Y. Bar-Ness, and U. Spagnolini, "Stable throughput of cognitive radios with and without relaying capability," IEEE Trans. on Commu., vol. 55, no. 12, pp. 2351-2360, Dec. 2007.

[3] J. Gambini, O. Simeone, and U. Spagnolini, "Cognitive relaying and opportunistic spectrum sensing in unlicensed multiple access channels," in IEEE 10th ISSSTA, Aug. 2008, pp. 371-375.

[4] M. Elsaadany, M. Abdallah, T. Khattab, M. Khairy, and M. Hasna, "Cognitive relaying in wireless sensor networks: Performance analysis and optimization," in IEEE GLOBECOM, Dec. 2010, pp. 1-6.

[5] S. Kompella, G. Nguyen, J. Wieselthier, and A. Ephremides, "Stable throughput tradeoffs in cognitive shared channels with cooperative relaying," in Proceedings IEEE INFOCOM, Apr. 2011, pp. 1961-1969.

[6] I. Krikidis, J. Laneman, J. Thompson, and S. McLaughlin, "Protocol design and throughput analysis for multi-user cognitive cooperative systems," IEEE Trans. on Wire. Commu., vol. 8, no. 9, pp. 4740-4751, Sept. 2009.

[7] A. El Shafie and A. Sultan, "Cooperative cognitive relaying with ordered cognitive multiple access," in GLOBECOM, Dec. 2012, pp. 1434-1439.

[8] I. Krikidis, N. Devroye, and J. Thompson, "Stability analysis for cognitive radio with multi-access primary transmission," IEEE Trans. on Wire. Commu., vol. 9, no. 1, pp. 72-77, Jan. 2010.

[9] X. Bao, P. Martins, T. Song, and L. Shen, "Stable throughput analysis of multi-user cognitive cooperative systems," in IEEE GLOBECOM, Dec. 2010, pp. 1-5

[10] A. Sadek, K. Liu, and A. Ephremides, "Cognitive multiple access via cooperation: protocol design and performance analysis," IEEE Trans. Inf. Theory, vol. 53, no. 10, pp. 3677-3696, Oct. 2007.

[11] R. Loynes, "The stability of a queue with non-independent inter-arrival and service times," in Proc. Cambridge Philos. Soc, vol. 58, no. 3. Cambridge University Press, 1962, pp. 497-520.

[12] W. Luo and A. Ephremides, "Stability of $\mathrm{N}$ interacting queues in random-access systems," IEEE Trans. Inf. Theory, vol. 45, no. 5, pp. 1579-1587, July 1999.

[13] R. Rao and A. Ephremides, "On the stability of interacting queues in a multiple-access system," IEEE Trans. Inf. Theory, vol. 34, no. 5, pp. 918-930, Sept. 1988. 